\title{
Spastic paraplegia-facial-cutaneous lesions syndrome
}

INSERM

\section{Source}

INSERM. (1999). Orphanet: an online rare disease and orphan drug data base. Spastic paraplegia-facial-cutaneous lesions syndrome. ORPHA:2819

Spastic paraplegia-facial-cutaneous lesions syndrome is a complex form of hereditary spastic paraplegia characterized by delays in motor development followed by a slowly progressive spastic paraplegia (affecting mainly lower extremities) associated with a desquamating facial rash with butterfly distribution (presenting at around two months of age) and dysarthria. There have been no further descriptions in the literature since 1982. 